during treatment while continued up to 14 months. Total daily doses of MPH in the combined treatment group were lower $(31 \mathrm{mg} / \mathrm{d})$ than in the medication alone group. (Jensen PS and MTA Cooperative Group. A 14-month randomized clinical trial of treatment strategies for attention-deficit/hyperactivity disorder. Arch Gen Psychiatry Dec 1999;56:1073-1086). (Respond: Peter S Jensen MD, Department of Child Psychiatry, Unit 78, NYS Psychiatric Institute, 1051 Riverside Dr, New York, NY 10032).

COMMENT. For the treatment of ADHD, carefully monitored methylphenidate is superior to behavioral therapy and to routine community care, that includes medication. Combined behavioral and stimulant therapies yield no greater benefits than medication alone for core ADHD symptom control, but may provide modest advantages for non-ADHD symptoms, including oppositional behavior and anxiety. One possible advantage of combining behavioral therapy with medication is the reduction in dose of MPH required and the consequent lessening of adverse effects. However, in practice the additional benefits of combined therapy are small and may not warrant the inconvenience and expense to the parents, except in patients who respond only partially to medication. The key to successful medical therapy is frequent monitoring and adjustment of dosage, not always achieved in clinical practice situations.

For a comment on the results of this study from an expert in the UK, see Taylor E. Development of clinical services for attention-deficit/hyperactivity disorder. Arch Gen Psychiatry Dec 1999;56:1097-1099. In the UK, the recognition and treatment of ADHD with medication is not as readily accepted as in the US. Many UK parents will opt for no therapy or behavioral therapy, despite the obvious benefits of MPH demonstrated in this MTA study.

ADHD Genetics. Association of the dopamine transporter gene (DAT1) with poor methylphenidate response is reported from Brookdale University Hospital, Brooklyn, NY (Winsberg BG, Comings DE. I Am Acad Child Adolesc Psychiatry Dec 1999;38:1474-1477). Homozygosity of the 10-repeat allele was found to characterize nonresponse to methylphenidate therapy.

\title{
GENETIC TRANSMISSION OF ADHD AND NOCTURNAL ENURESIS
}

The transmission of primary nocturnal enuresis (PNE) in relatives of PNE and control probands, with and without $\mathrm{ADHD}$, was examined at the University of California at Los Angeles. Among 126 male probands with and without PNE and ADHD, ages 6-12 yrs, and 338 of their first-degree relatives (237 parents and 101 siblings), the rates of enuresis in parents of enuretic children (40\%) were significantly higher than in parents of non-enuretic control children (6\%), regardless of the co-morbid ADHD of the proband. Of the PNE probands $65 \%$ had at least one affected parent, and of these families, $24 \%$ had an affected sibling. The rates of PNE between parents or siblings in the PNE and PNE + ADHD groups were similar, both groups having significantly elevated rates of PNE in their relatives. In the ADHD only group, there was no familial clustering of PNE. The rate of PNE in first-degree relatives is independent of ADHD in the proband. ADHD and PNE are transmitted independently. (Bailey JN, Ornitz EM, Gehricke JG, Gabikian P, Russell AT, Smalley SL. Transmission of primary nocturnal enuresis and attention deficit hyperactivity disorder. Acta Paediatr Dec 1999;88:1364-1368). (Respond: JN Bailey, University of California at Los Angeles, 760 Westwood Place Box 63, Los Angeles, CA 90024).

COMMENT. The familial nature of nocturnal enuresis is suggested by family 
studies, twin studies, and a high rate of positive family histories in case studies. The risk of PNE to parents is 6.7 -fold, and the risk to siblings 3.6-fold. Rates of enuresis in individuals with ADHD range from 20-30\%, compared to $10 \%$ in the average population of $7 \mathrm{yr}$ old children. In both conditions, boys are affected more frequently than girls. The frequency of ADHD is increased in PNE children compared to non-PNE controls. The present study suggests that the association of PNE and ADHD is not due to a shared genetic basis.

Factors in etiology of PNE other than genetic transmission are also considered in a commentary by Jarvelin M-R (Acta Paediatr Dec 1999;88:13151317). These include delayed maturation of the nervous system, disturbed arginine-vasopressin secretion, prenatal and birth-related factors, neurobehavioral disorders, and psychosocial factors. Many of these factors also apply to the presumed etiology of ADHD.

\section{META-ANALYSIS STUDY OF CLONIDINE IN ADHD}

The literature on the clinical use of clonidine for attention deficit hyperactivity disorder (ADHD), from 1980-1999, was reviewed at the Department of Psychiatry, University of Massachusetts Medical School, Worcester, MA. In 11 studies analyzed clonidine had positive effects in the treatment of ADHD and ADHD comorbid with conduct disorder, developmental delay, and tic disorder. The benefits were reported by clinician, parent, and teacher, and the effect was of moderate size $(0.58+/-0.16)$ and less than that of stimulants. The effect was significantly greater for the ADHD-alone group. Clonidine was associated with a high prevalence of side effects, especially sedation, irritability, night awakening, hypotension, and dizziness. Two studies using the transdermal system reported a high percentage of localized rash, erythema, and skin irritation under the patch. In one of 3 studies with ECG data, changes were noted but no cardiac symptoms. (Connor DF, Fletcher KE, Swanson JM. A meta-analysis of clonidine for symptoms of attention-deficit hyperactivity disorder. I Am Acad Child Adolesc Psychiatry Dec 1999;38:1551-1559). (Reprints: Dr Daniel F Connor, Department of Psychiatry, Room S7828 , University of Massachusetts Medical School, Worcester, MA 01655).

COMMENT. Clonidine is a second line treatment for ADHD and is less effective than stimulants, even in patients with comorbid disorders. A moderate size benefit is associated with a high incidence of side effects. In combination with stimulants, the risk of serious side effects is a concern, and a treatment to be avoided pending further study. The disappointing results of this meta-analysis may promote a greater interest in Tenex as an alternative treatment for ADHD children with comorbidity, including tics, insomnia, and oppositional defiance disorder.

\section{PANDAS, TICS AND OCD, AND IMMUNOTHERAPY}

Thirty children with severe, streptococcal-triggered exacerbations of tics or obsessive compulsive disorder $(O C D)$ were treated with plasma exchange (5 in 2 weeks), intravenous immunoglobulin (IVIG, $1 \mathrm{~g} / \mathrm{kg}$ daily on 2 consecutive days). or placebo saline solution, randomly assigned, in a study at the National Institutes of Health, Bethesda, MD. Neuropsychiatric medications were continued through the study, and oral penicillin or erythromycin was given during follow-up to protect against streptococcal infections. Throat cultures were negative at baseline, and antistreptolysin-O titers were positive in at least half the patients in each treatment group (mean 350-517). Similar elevations were found for AS deoxyribonucleic B titers. At 1 month, the improvements in the IVIG and plasma exchange groups were $45-58 \%$ for $O C D, 31-47 \%$ for anxiety, 33-35\% for overall 